\title{
A new nearby pulsar wind nebula overlapping the RX J0852.0-4622 supernova remnant
}

\author{
F. Acero ${ }^{1,2}$, Y. Gallant ${ }^{1}$, J. Ballet $^{3}$, M. Renaud ${ }^{1}$, and R. Terrier ${ }^{4}$ \\ ${ }^{1}$ LUPM, CNRS/Université Montpellier 2, France \\ e-mail: facero@in2p3.fr \\ 2 NASA Goddard Space Flight Center, Greenbelt, MD 20771, USA \\ 3 AIM, CEA/CNRS/Université Paris Diderot-Paris 7, France \\ 4 APC, CNRS/Université Paris Diderot-Paris 7, France
}

Received 27 November 2012 / Accepted 17 December 2012

\section{ABSTRACT}

\begin{abstract}
Context. Energetic pulsars can be embedded in a nebula of relativistic leptons that is powered by the dissipation of the rotational energy of the pulsar. The object PSR J0855-4644 is an energetic and fast-spinning pulsar $\left(\dot{E}=1.1 \times 10^{36} \mathrm{erg} \mathrm{s}^{-1}, P=65 \mathrm{~ms}\right)$ discovered near the southeast rim of the supernova remnant (SNR) RX J0852.0-4622 (aka Vela Jr) by the Parkes multibeam survey. The position of the pulsar is in spatial coincidence with an enhancement in X-rays and $\mathrm{TeV} \gamma$-rays, which could be due to its putative pulsar wind nebula (PWN).

Aims. The purpose of this study is to search for diffuse non-thermal X-ray emission around PSR J0855-4644 to test for the presence of a PWN and to estimate the distance to the pulsar.

Methods. An X-ray observation was carried out with the XMM-Newton satellite to constrain the properties of the pulsar and its nebula. The absorption column density derived in X-rays from the pulsar and from different regions of the SNR rim was compared with the absorption derived from the atomic $(\mathrm{HI})$ and molecular $\left({ }^{12} \mathrm{CO}\right)$ gas distribution along the corresponding lines of sight to estimate the distance to the pulsar and to the SNR.

Results. The observation has revealed the X-ray counterpart of the pulsar together with surrounding extended emission, thus confirming the existence of a PWN. The comparison of column densities provided an upper limit to the distance to the pulsar PSR J0855-4644 and the SNR RX J0852.0-4622 ( $d \leq 900 \mathrm{pc})$. Although both objects are at compatible distances, we rule out that the pulsar and the SNR are associated. With this revised distance, PSR J0855-4644 is the second most energetic pulsar, after the Vela pulsar, within a radius of $1 \mathrm{kpc}$ and could therefore contribute to the local cosmic-ray $\mathrm{e}^{-} / \mathrm{e}^{+}$spectrum.
\end{abstract}

Key words. ISM: supernova remnants - pulsars: general - X-rays: individuals: RX J0852.0-4622

\section{Introduction}

The Vela region (approximately centered on $\ell=265^{\circ}, b=-1^{\circ}$ ) is host of several remnants of stellar explosions including the eponymous supernova remnant (SNR). The Vela remnant is among the brightest and most extended X-ray sources in the sky and is also the closest SNR currently detected (the distance to the remnant's pulsar has been measured to be $290 \mathrm{pc}$; Dodson et al. 2003). With its large angular size ( $8^{\circ}$ in diameter) the object is overlapping several remnants such as the Puppis A SNR and RX J0852.0-4622. In the X-ray spectral domain, this complex region is dominated by the Vela and Puppis remnants in soft X-rays and the RX J0852.0-4622 SNR (aka Vela Jr) becomes apparent only above $1 \mathrm{keV}$ (Aschenbach 1998).

The Parkes multibeam radio survey discovered a pulsar, PSR J0855-4644, lying on the southeast rim of the RX J0852.0-4622 SNR (Kramer et al. 2003). This young and energetic pulsar (characteristic age $\tau_{\text {c }}=140$ kyrs, $\dot{E}=1.1 \times$ $10^{36} \mathrm{erg} \mathrm{s}^{-1}$ and $P=65 \mathrm{~ms}$ ) is in spatial coincidence with an enhancement in the X-and $\gamma$-ray maps (presented in Aschenbach et al. 1999; Aharonian et al. 2007, respectively), which could be the signature of its pulsar wind nebula (PWN). Based on the radio dispersion measure of the pulsar $\left(\mathrm{DM}=239 \mathrm{~cm}^{-3} \mathrm{pc}\right.$; Kramer et al. 2003) combined with the NE2001 model of Galactic electron distribution from Cordes \& Lazio (2002), the distance to the pulsar is estimated to be $4 \mathrm{kpc}$. However, estimating the distance with this method in this specific region of the sky can be erroneous (see Mitra \& Ramachandran 2001) due to the structured and nearby emission of the Gum nebula, a $35^{\circ}$ large nebula located at $\sim 500 \mathrm{pc}$. The electron density inside the Gum nebula can strongly vary from 0.1 to $100 \mathrm{~cm}^{-3}$ (Reynolds 1976; Wallerstein et al. 1980; Dubner et al. 1992; Reynoso \& Dubner 1997) and therefore so can the DM of a pulsar lying in the background of the nebula. This complex region is modeled as a simple sphere (the Gum nebula) of homogenous density in the NE2001 model.

To better constrain the distance to the pulsar and search for a potential X-ray nebula, an XMM-Newton observation was carried out, whose results are presented in this article. In Sect. 2, we present a detailed morphological and spectral analysis of the pulsar and its nebula. A discussion of the distance to the PSR and to the SNR RX J0852.0-4622 is presented in Sect. 3. To conclude, a summary of the proposed scenario, the potential relation between PSR J0855-4644 and the RX J0852.0-4622 SNR and the expected $\mathrm{TeV}$ emission from the PWN is presented in Sect. 4.

\section{X-ray observations}

The SNR RX J0852.0-4622 was first discovered in X-rays by the ROSAT satellite in a high-energy image $(E>1.3 \mathrm{keV})$ and 


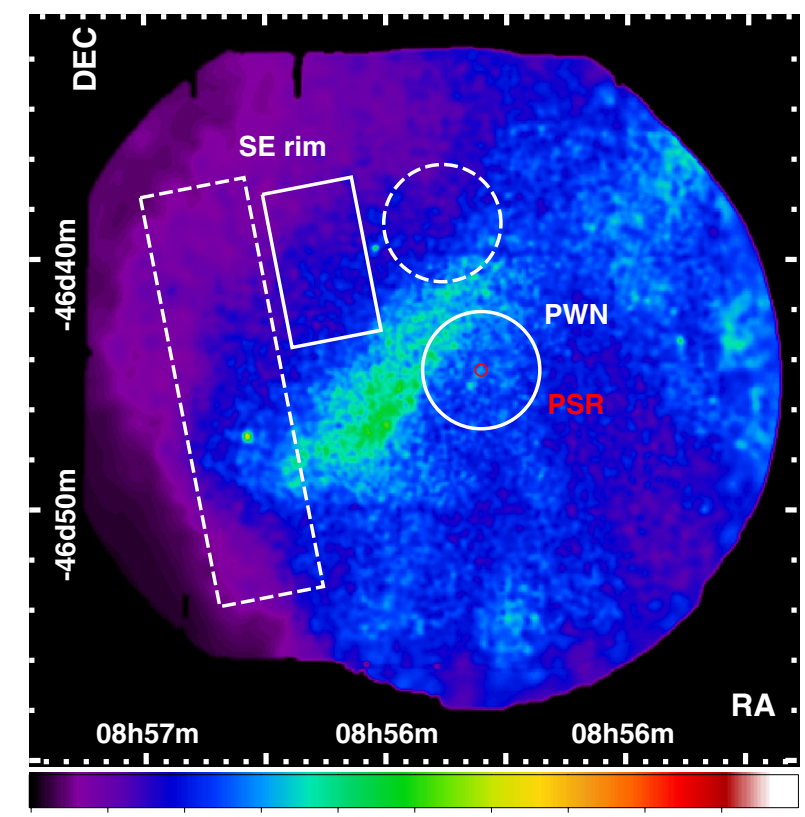

0

4E-05

8E-05

0.00012

0.00016

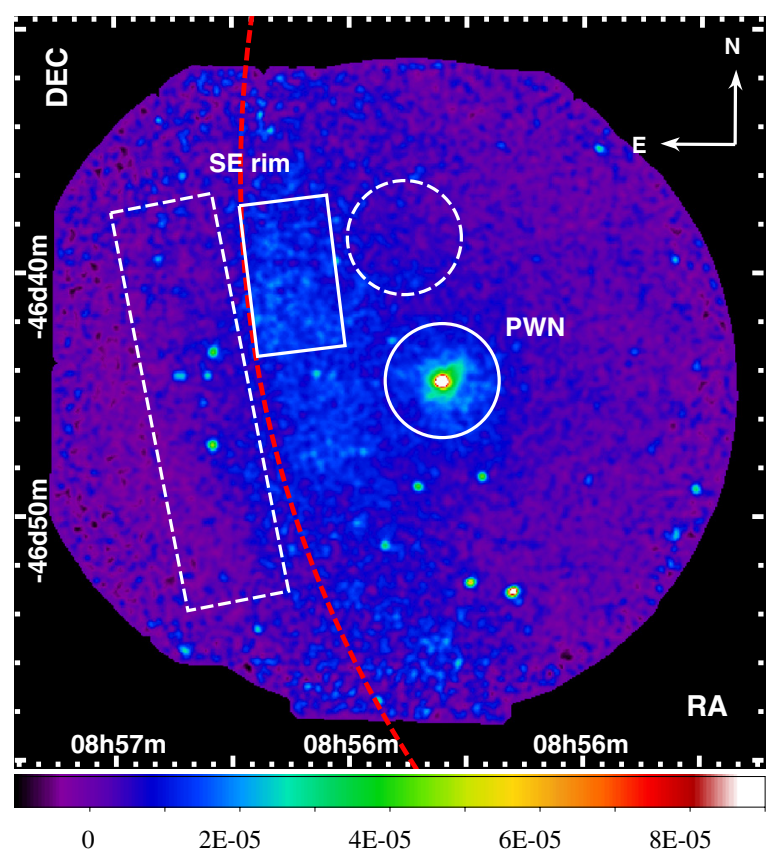

Fig. 1. XMM-Newton background-subtracted flux images combining MOS and PN cameras in units of $\mathrm{ph} / \mathrm{cm}^{2} / \mathrm{s} / \mathrm{arcmin}^{2}$. Left: low-energy band image (0.5-0.8 keV) adaptively smoothed to a signal-to-noise ratio of 10 using the task asmooth provided in the XMM-Newton software SAS. The position of PSR J0855-4644 is shown with a red circle. Right: image in the high-energy band (1.2-6 keV) smoothed with a Gaussian width of 7". The dashed red line shows approximately the SNR shell RX J0852.0-4622. In solid line we show the spectral extraction regions used to study the rim of RX J0852.0-4622 and the PWN whereas the dashed line regions are used to estimate the spectrum of the astrophysical background.

has revealed the energy-dependent morphology of this complex region. Due to its large angular size (nearly $1^{\circ}$ in radius), the SNR has only been partly observed by the new generation of $\mathrm{X}$-ray telescopes and, so far, no mosaics that cover the whole remnant at high angular resolution is available. Most observations have been focused on the intriguing bright and thin filaments that emit synchrotron emission in the northwest of the remnant (see e.g. Bamba et al. 2005).

The XMM-Newton observation presented in this study is centered on the pulsar and also unveils the previously unobserved southeastern shell of the RX J0852.0-4622 SNR. Several other $X M M-N e w t o n$ observations in the northwest and in the south of the SNR were used in this study to compare the spectral properties of the SNR along the shell.

\subsection{Data analysis}

The region of the pulsar was observed with XMM-Newton for $55 \mathrm{ks}$ using the Full Frame mode of the three EPIC instruments. After flare screening, the remaining exposure time is $40 \mathrm{ks}$ and $29 \mathrm{ks}$ for the MOS and PN cameras, respectively. For the image generation, the instrumental background was derived from a compilation of blank sky observations (Carter \& Read 2007) renormalized in the $10-12 \mathrm{keV}$ energy band. The background-subtracted and vignetting-corrected images in a low $(0.5-0.8 \mathrm{keV})$ and high-energy band (1.2-6 keV) are presented in Fig. 1. These images clearly show two very different facets of the Vela region. While the low-energy image is dominated by the large scale structure of thermal emission from the Vela SNR, the high-energy band reveals a faint rim of the RX J0852.0-4622 SNR and the X-ray counterpart of the pulsar. Interestingly, diffuse emission is also seen surrounding the pulsar. The spectral properties of the pulsar and the diffuse emission are presented in the following sections.
The thermal emission from the Vela SNR is extremely bright at low-energy $(E<1 \mathrm{keV})$ and therefore the statistics gathered with the PN camera is very high. We have encountered a calibration problem with the spectral response of the PN camera, particularly visible in the oxygen lines. This is a known problem for the XMM-Newton team, and following their recommendation, an offset in energy in the PN spectral response function $(\mathrm{rmf})$ was introduced via the gain function in Xspec. See Appendix A for more details. All spectra were fitted using MOS1+2 and PN, although for graphical purposes, only the MOS $1+2$ spectra are shown along with the best-fit obtained with the three instruments.

\subsection{Morphology of the nebula}

To further investigate the morphology of this diffuse emission, a radial profile (shown in Fig. 2, middle), centered on the position of the pulsar, was extracted in the high-energy band $(1.2-6 \mathrm{keV})$. The profile was then fitted with a combination of a point-like component (using the XMM-Newton point spread function (PSF) from the calibration files), an extended component (a Gaussian profile), and a constant that represent the pulsar, the nebula, and the background. The best-fit Gaussian width deconvolved from the PSF of the extended component is $45^{\prime \prime} \pm 1.5^{\prime \prime}$ (unless otherwise noted, all error bars are given at a $90 \%$ confidence level) and the diffuse emission around the pulsar is detected up to $\sim 150^{\prime \prime}$. The large-scale emission is fairly symmetric and no indications of a trail or of a bow-shock structure (reminiscent of a fast-moving pulsar) are observed.

Interestingly, at smaller scale the inner part of the nebula $\left(r<60^{\prime \prime}\right)$ seems to show some azimuthal variation. To test the significance of these variations, an azimuthal profile was extracted from an annulus region $\left(\mathrm{R} 1=15^{\prime \prime}<r<\mathrm{R} 2=60^{\prime \prime}\right.$, see Fig. 2 top panel for region definition). The resulting brightness 

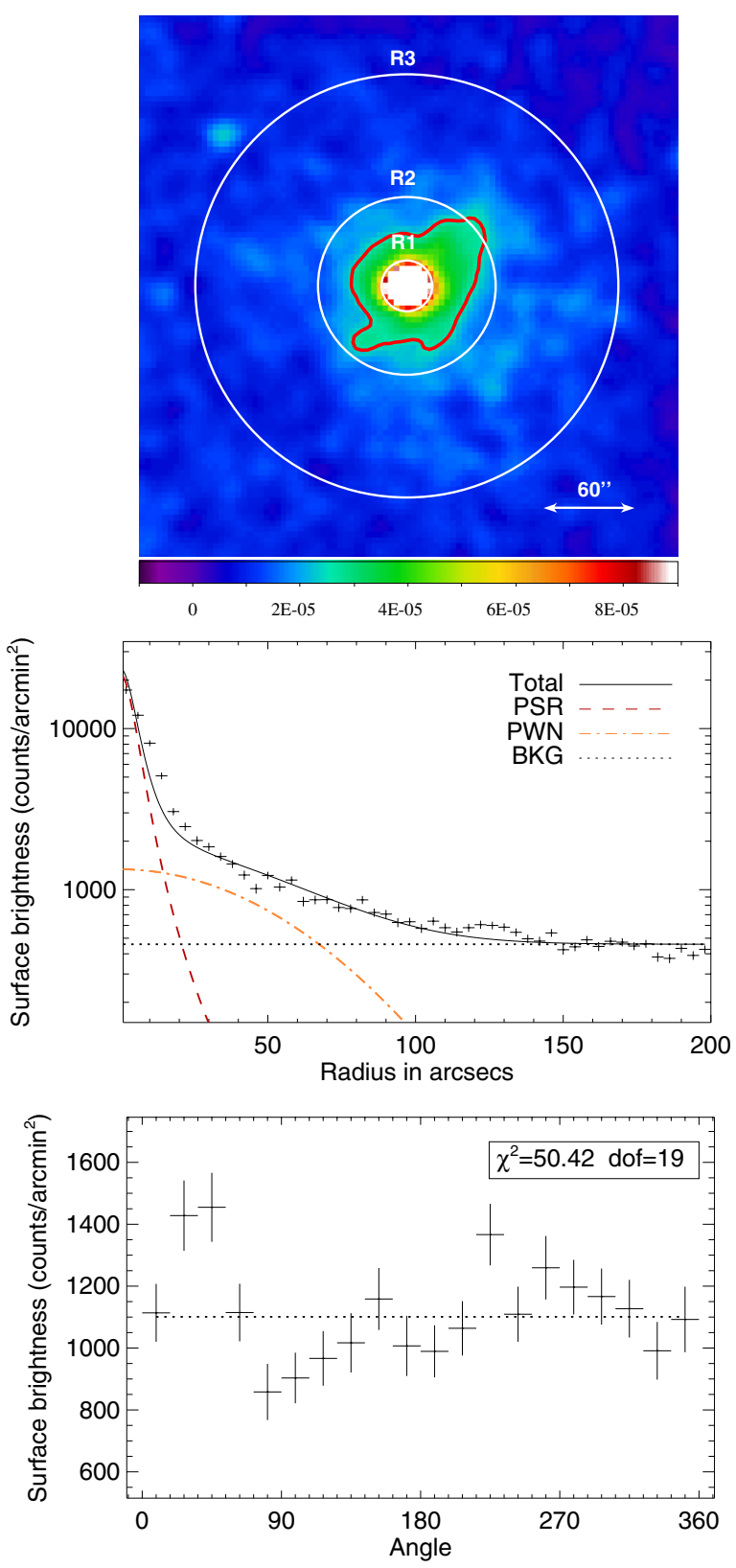

Fig. 2. Top: zoom on the PWN region in the 1.2-6 keV image shown in Fig. 1 (right). North is up and east to the left. The region is annotated with the annulus regions of radii R1, R2, and R3 (15", 60", and $\left.150^{\prime \prime}\right)$ used in the morphological and spectral analysis. A contour at the level of $2.5 \times 10^{-5} \mathrm{ph} / \mathrm{cm}^{2} / \mathrm{s} / \mathrm{arcmin}^{2}$ is overlaid in red to outline the morphology of the inner nebula. Middle: radial profile of the PWN in the high-energy band (1.2-6 keV) extracted from a region centered on the pulsar. The best-fit two-components model (point-like + diffuse) is overlaid. Bottom: azimuthal profile extracted from the annulus region R1-R2 $\left(15^{\prime \prime}<r<60^{\prime \prime}\right)$ illustrated in the top panel in the high-energy band. Position angle $0^{\circ}$ corresponds to the west and $90^{\circ}$ to the north. Two significant enhancements over the mean value (dashed line) are seen at $\sim 50^{\circ}$ and $\sim 230^{\circ}$. The fact that these knots of emission are separated by $180^{\circ}$ is suggestive of a jet morphology.

distribution presented in Fig. 2 (bottom) is not compatible with a flat profile and exhibits features, $180^{\circ}$ apart, at $50^{\circ}$ and $230^{\circ}$ that are suggestive of jet-like structures as observed in many other young pulsars (see Kargaltsev \& Pavlov 2008). However, the limited spatial resolution of XMM-Newton hampers a study of the immediate environment of the pulsar. To confirm the presence of jets in the nebula and to search for a torus, high spatial resolution observations with the Chandra X-ray satellite would be needed.

\subsection{Spectral analysis of the nebula}

To extract the spectral properties of the nebula, the instrumental and astrophysical backgrounds have to be modeled carefully in this complicated region of the sky. The instrumental- and particle-induced background were derived from a compilation of observations with the filter wheel in closed position ${ }^{1}$ and renormalized in the 10-12 keV energy band over the whole field of view. The spectral data were fitted using Cash statistics and the goodness-of-fit was assessed trough Monte Carlo simulations using the goodness command in Xspec (v12.5). This command returns the fraction of simulated spectra whose fit statistics value is lower than the one obtained on the real data, which is equivalent to $1-p-$ value $^{2}$. If the model is a good representation of the data, a $p$-value around 0.5 is expected. If the $p$-value is close to 0 , the model is clearly a poor representation of the data. The Tuebingen-Boulder ISM absorption model (tbabs) was used for all absorbed components together with the abundances of Wilms et al. (2000).

The astrophysical background in this region is particularly complex, as illustrated in Fig. 1. The low-energy part of the spectrum is dominated by the thermal emission from the Vela SNR and from the Local Bubble, while in the high-energy band nonthermal emission from RX J0852.0-4622 (the contribution from the SNR's rim at the PWN's position is not negligible; see Sect. 2.7) and the cosmic X-ray background (CXB) are present. To model this background a spectrum from a region (dashed circle in Fig. 1, right) located at the same distance from the shock front of the RX J0852.0-4622 SNR (to have similar spectral properties) was obtained.

The spectrum from this background region is well fitted by a two-component model ( $p$-value $=0.24)$. The first component is an absorbed variable abundances astrophysical plasma emission code (VAPEC) model that represents the thermal contribution from Vela. The X-ray absorption column $N_{\mathrm{H}}$ of this component is poorly constrained with XMM-Newton's energy range $(0.4-10 \mathrm{keV})$ and was therefore fixed to $5 \times 10^{20} \mathrm{~cm}^{-2}$, the typical value derived by Aschenbach et al. (1995) using data from the ROSAT satellite, whose energy range extends to lower energies (0.1-2.4 keV). The best-fit parameters for this thermal component are the following: $k T=0.14 \mathrm{keV}$, oxygen abundance $=0.45^{3}$, and a normalization of $7.1 \times 10^{-3} \mathrm{~cm}^{-5}$. The second component is an absorbed power-law model representing the contributions from RX J0852.0-4622 and the CXB. Adding another component to separately represent the CXB, with spectral parameters fixed from De Luca \& Molendi (2004), did not improve the quality of the fit. The best-fit parameters give $N_{\mathrm{H}}=(0.45 \pm 0.10) \times 10^{22} \mathrm{~cm}^{-2}, \Gamma=1.90 \pm 0.04$, and norm $=(2.70 \pm 0.11) \times 10^{-4} \mathrm{keV}^{-1} \mathrm{~cm}^{-2} \mathrm{~s}^{-1}$ at $1 \mathrm{keV}$.

The best-fit model obtained from the background spectrum was then used as a fixed template model in the spectrum of the nebula (annulus: $\mathrm{R} 1=15^{\prime \prime}<r<R 3=150^{\prime \prime}$ ) where the

\footnotetext{
http://xmm2.esac.esa.int/external/xmm_sw_cal/ background/filter_closed/

2 The $p$-value is the probability of obtaining a fit statistic higher than the observed value.

3 The abundance of neon was also let to free to vary as a test, but was found to be compatible with the solar abundance and was therefore fixed to the solar value in the model.
} 

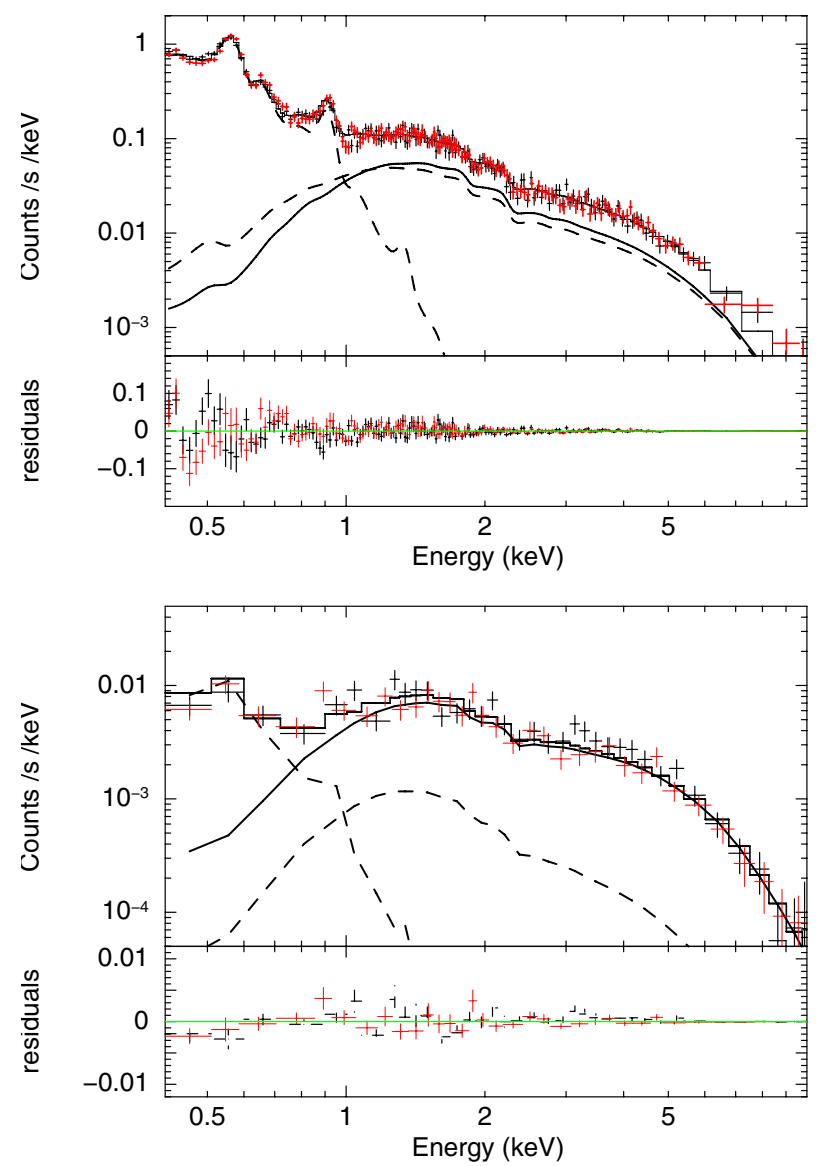

Fig. 3. Top: X-ray spectrum of the nebula extracted from a $15-150^{\prime \prime}$ annulus region shown in Fig. 1. The astrophysical background components (dominated by thermal emission from Vela + non-thermal emission from RX J0852.0-4622) are depicted by dashed lines while the non-thermal emission of the nebula is illustrated by the solid line. Bottom: X-ray spectrum of PSR J0855-4644 extracted from a 15" radius region. The astrophysical background (dashed lines) is composed of Vela's thermal emission at low energies while the contribution of the nebula and of RX J0852.0-4622 at higher energies is not negligible. For graphical purposes only the MOS1 and MOS2 data are shown although the PN data were also used in the fitting process.

normalization was set to the ratio of geometrical areas for the non-thermal emission and was let free to vary for the thermal emission because the emission from the Vela SNR is seen to slightly vary in the low-energy map shown in Fig. 1. All other parameters ( $k T$, power-law absorption column, and spectral index) for the background model were kept fixed. To represent the emission from the nebula, an absorbed power-law model was added to the previously derived background model. The spectrum from the nebula is well modeled (see Fig. 3, top) with the sum of the background + power-law model $(p$-value $=0.14)$, thus confirming the non-thermal nature of the nebula's X-ray emission.

\subsection{Spectral analysis of PSR J0855-4644}

To derive the X-ray properties of the pulsar, and in particular the column density along the line of sight to discuss its distance, we extracted a spectrum in a $15^{\prime \prime}$ radius region centered on the pulsar. For this region, the astrophysical background is composed of Vela and the Local Bubble at low energies and of the sum of the contributions from the nebula (which is not negligible based on the radial profile modeling in Fig. 2), RX J0852.0-4622 and the CXB.

The astrophysical background model was obtained from the best-fit model of the spectrum extracted in the PWN region $\left(15^{\prime \prime}<r<150^{\prime \prime}\right)$. The non-thermal component of the template was renormalized using the nebula's geometrical model shown in Fig. 2 (middle) while the thermal component was renormalized using the geometrical ratio of the areas (i.e., thermal emission is assumed to be homogeneous at the scale of the PWN).

The spectrum of the pulsar (presented in Fig. 3, bottom) is well described $(p$-value $=0.46)$ at low energies by the fixed template (absorbed VAPEC model) and at high energies with two absorbed power-laws, one representing the astrophysical background and one for the non-thermal emission of the pulsar. The spectral parameters of the pulsar best-fit model are listed in Table 1. A black body model has also been tested to model the $\mathrm{X}$-ray emission of the pulsar, but resulted in a poor fit to the data $\left(p\right.$-value $\left.=4 \times 10^{-3}\right)$.

The derivation of the $N_{\mathrm{H}}$ from the pulsar is sensitive to the normalization of the thermal component, dominating at low energies. To estimate the accuracy to which we can fix the normalization of the thermal model, we calculated the ratio of brightnesses in the pulsar region over the PWN region in the $0.4-0.6 \mathrm{keV}$ energy band. The resulting ratio is $1.13 \pm 0.08$, confirming that the assumption of homogeneous thermal emission at the scale of the PWN is reasonable. When the normalization of the thermal component in the pulsar spectral model is increased by a factor 1.13 , the best-fit value of $N_{\mathrm{H}}$ increases to $0.71_{-0.11}^{+0.13} \times 10^{22} \mathrm{~cm}^{-2}$, within statistical errors of the value quoted in Table 1. This test provides an estimate of the systematic error associated with the normalization of the thermal emission at low energies.

\subsection{Electron cooling in the nebula}

To test for a possible spectral cooling in the nebula, a spectrum was derived in the inner $\left(15^{\prime \prime}<r<60^{\prime \prime}\right)$ and outer part of the nebula $\left(60^{\prime \prime}<r<150^{\prime \prime}\right)$. For both regions, the astrophysical background was fixed to the best-fit model obtained from the whole PWN in Sect. 2.3. To limit the degeneracy between the absorption column and the photon index, the $N_{\mathrm{H}}$ of non-thermal emission from the nebula was fixed to the value derived from the pulsar.

The best-fit parameters of the inner and outer region of the nebula are presented in Table 1. A significant steepening of the spectral index toward the external region of the nebula is observed (from $1.70_{-0.06}^{+0.07}$ to $2.01_{-0.04}^{+0.04}$ ). This is consistent with the scenario of a population of electrons cooling as they are advected away from the pulsar.

In connection with the spectral parameter evolution in the inner nebula, the background model for the pulsar was revisited. The inner region of the nebula, instead of the whole nebula, was tested as the template background model for the pulsar. The best-fit parameters of the pulsar with this new background model are $N_{\mathrm{H}}=0.59_{-0.13}^{+0.14} \times 10^{22} \mathrm{~cm}^{-2}$ and Index $=1.20_{-0.11}^{+0.08}$. When rescaling the normalization of the thermal component by the same 1.13 factor (as tested in the previous section), the $N_{\mathrm{H}}$ value slightly increases to $0.67_{-0.13}^{+0.15} \times 10^{22} \mathrm{~cm}^{-2}$ and Index $=1.23_{-0.11}^{+0.10}$. Those parameters are compatible with those presented in Table 1, and in the following discussion only the best-fit parameters from the pulsar presented in the table are used. 
Table 1. Best-fit parameters obtained on the nebula, the pulsar, and different parts of the rim of the RX J0852.0-4622 SNR.

\begin{tabular}{lccccc}
\hline \hline & $N_{\mathrm{H}}$ & $\begin{array}{c}\text { Power-law best-fit parameters } \\
\text { index }\end{array}$ & flux $\left(10^{-12} \mathrm{ergs} / \mathrm{cm}^{2} \mathrm{~s}^{-1}\right)$ & $\begin{array}{c}\text { Region area } \\
\text { arcmin }\end{array}$ & $\begin{array}{c}\text { Annulus radii } \\
\text { arcsec }\end{array}$ \\
\hline Pulsar $\mathcal{F}$ PWN & & & & & \\
PSR J0855-4644 & $0.64_{-0.11}^{+0.13}$ & $1.24_{-0.10}^{+0.09}$ & $0.26_{-0.05}^{+0.03}$ & 0.2 & $0-15$ \\
PWN & $0.76_{-0.05}^{+0.06}$ & $2.03_{-0.04}^{+0.06}$ & $0.88_{-0.08}^{+0.04}$ & 19.4 & $15-150$ \\
Inner PWN & 0.64 & $1.70_{-0.06}^{+0.07}$ & $0.39_{-0.06}^{+0.02}$ & 2.9 & $15-60$ \\
Outer PWN & 0.64 & $2.01_{-0.04}^{+0.04}$ & $0.55_{-0.06}^{+0.03}$ & 16.5 & $60-150$ \\
\hline SNR & & & & & \\
Southeast rim & $0.88_{-0.06}^{+0.08}$ & $2.32_{-0.08}^{+0.09}$ & $1.83_{-0.09}^{+0.08}$ & 25.7 & \\
South rim & $0.85_{-0.05}^{+0.05}$ & $2.66_{-0.07}^{+0.05}$ & $2.88_{-0.08}^{+0.09}$ & 42.1 & \\
Northwest rim & $0.68_{-0.05}^{+0.04}$ & $2.53_{-0.04}^{+0.05}$ & $4.10_{-0.13}^{+0.11}$ & 15.2 & \\
\hline
\end{tabular}

Notes. For each spectrum, a two-components (absorbed VAPEC + absorbed power-law, see Sect. 2.3 for details) astrophysical background model was estimated from the background regions shown in Figs. 1 and 4. Only the parameters from the non-thermal components are listed in this table while the parameters of the thermal component are fairly constant from one region to another $\left(k T=0.14-0.17 \mathrm{keV}\right.$ and the $N_{\mathrm{H}}$ was fixed to $5 \times 10^{20} \mathrm{~cm}^{-2}$ for all the regions). The unabsorbed non-thermal fluxes of the PWN, PSR and SNR's rim are given in the 2-10 keV energy range and the $N_{\mathrm{H}}$ in units of $10^{22} \mathrm{~cm}^{-2}$. The area of the extraction region and the annulus radii for the PSR/PWN analysis are also given. The error bars are given at $90 \%$ confidence level.

\subsection{Spectral analysis of the SNR rim}

To discuss the relative distances of the SNR and of the PWN, we carried out a spectral analysis of different regions of the RX J0852.0-4622 SNR. In the same pointing where the PWN is observed, we extracted a spectrum from the rim in the region labeled SE rim in Fig. 1. The region does not fully encompass the rim to avoid the bright structure of thermal emission from Vela that would hamper a proper determination of the absorption column. As in the previous sections, an astrophysical background model (absorbed VAPEC + absorbed power-law) was fitted to a region outside the SNR represented by the dashed box in Fig. 1. The total spectral model for the SNR rim is the sum of the previously estimated background model plus an absorbed power-law representing the synchrotron emission from the SNR. The parameters of the background model were fixed in the analysis of the rim except the normalization of the thermal emission. The best-fit parameters of the non-thermal emission from the rim are listed in Table 1. The same procedure was then applied to other regions of the SNR where X-ray observations were available, namely the northwest and the south of the SNR. The $1.2-6 \mathrm{keV}$ image of those regions together with the spectral extraction regions and background regions are shown in Fig. 4. The eastern rim of the SNR has also been observed by XMM-Newton but the observation has been, unfortunately, strongly affected by solar flares and only $5 \mathrm{ks}$ out of $36 \mathrm{ks}$ of observation are usable. As a consequence, no reliable spectral information could be derived from the diffuse and faint emission from the SNR in this region.

The resulting best-fit parameters of the spectral analysis from three different regions of the SNR are listed in Table 1.

\subsection{Radial profiles of the SNR rim}

To estimate the non-thermal contribution of RX J0852.0-4622 at the PWN position, a radial profile was extracted from a region of the same width and direction as the region labeled SE rim in Fig. 1 (right panel) but with a different length. The resulting profile, shown in Fig. 5 (top), demonstrates that the emission of RX J0852.0-4622 is not negligible at the reported distance of the PWN behind the shock front and its implementation in the astrophysical background modeling, in Sect. 2.3, is justified.
To measure the scale width of this filament, the profile was fitted with an exponentially decreasing model of the form $\mathrm{e}^{-\left|r_{0}-r\right| / w_{d}}$, where $d$ denotes the downstream side of the shock (same geometrical model as in Bamba et al. 2005, where the northwest rim of the SNR is studied). The best-fit model is overlaid in Fig. 5 (top) and the best-fit parameter is $w_{d}=6.85 \pm$ $0.55 \mathrm{arcmin}$. For comparison, the radial profile of the northwestern region is shown in Fig. 5 (bottom). The latter profile was obtained from a region of the same direction as the white solid region shown in Fig. 4 (top). The same geometrical model was used and the best-fit parameter is $w_{d}=1.61 \pm 0.05$ arcmin. We note that the downstream scale of the rim in this region is nearly four times thinner than in the southeast region. The implication of those different widths on the value of the magnetic field that can be derived is discussed in more detail in Sect. 4.5.

\section{Distance estimate}

The method for tackling the problem of the distance to the pulsar is based on the existence of a strong contrast of integrated ${ }^{12} \mathrm{CO}$ between the eastern and the western parts of the SNR, as shown in Fig. 7 (left). This contrast is due to the Vela molecular ridge (VMR) cloud C (see Murphy \& May 1991, for cloud nomenclature), lying at a distance of $700 \pm 200 \mathrm{pc}$. One of the method to derive this distance is an infrared photometric study of the star-forming regions in the VMR (see Liseau et al. 1992, and references therein). Other independent methods, discussed in Murphy \& May (1991), give a similar distance. This cloud is the main contributor of ${ }^{12} \mathrm{CO}$ emission along the line of sight, as seen in Fig. 6 (bottom panel), and is located at a radial velocity in the local standard of rest of $V_{\mathrm{LSR}} \sim 5 \mathrm{~km} \mathrm{~s}^{-1}$. If the SNR RX J0852.0-4622 lies behind the VMR, the observed gradient in ${ }^{12} \mathrm{CO}$ should also be visible in the measurement of the $\mathrm{X}$-ray absorption column.

To investigate this correlation, absorption column density maps (presented in Fig. 7) were calculated using HI data from the Parkes Galactic all-sky survey (McClure-Griffiths et al. 2009; Kalberla et al. 2010) and ${ }^{12} \mathrm{CO}$ data from the Dame et al. (2001) survey. To fully enclose the gas material lying between the observer and the back of the VMR, the data were integrated on radial velocities from $-10 \mathrm{~km} \mathrm{~s}^{-1}$ to $15 \mathrm{~km} \mathrm{~s}^{-1}$. Due to the intrinsic velocity dispersion of the ${ }^{12} \mathrm{CO}$ emission line, 


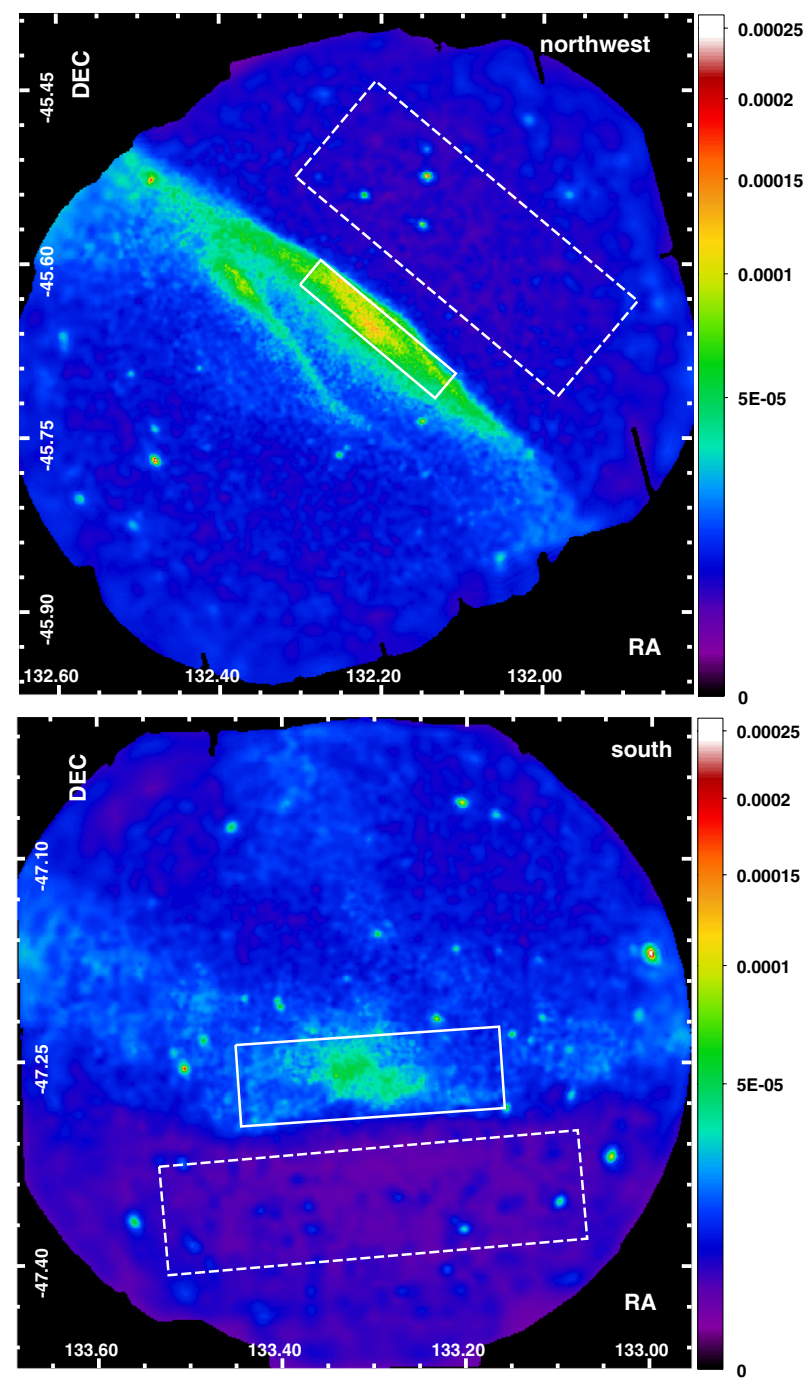

Fig. 4. XMM-Newton background-subtracted flux images (combining MOS and PN cameras) of the northwest (top) and south (bottom) pointings of RX J0852.0-4622 in the 1.2-6 keV energy band. As in Fig. 1, the solid lines illustrate the regions of spectral extraction, whereas the dashed lines represent the regions used to estimate the astrophysical background. The images were adaptively smoothed to reach a $\mathrm{S} / \mathrm{N}$ of 10 , the color scale is in square root and the units are $\mathrm{ph} / \mathrm{cm}^{2} / \mathrm{s} / \operatorname{arcmin}^{2}$.

it is unfortunately not possible to estimate the column density between the observer and the foreground of the VMR. The COto- $\mathrm{H}_{2}$ mass conversion factor and the $\mathrm{HI}$ brightness temperature to $N_{\mathrm{H}}$ used to produce the maps are $1.8 \times 10^{20} \mathrm{~cm}^{-2} \mathrm{~K}^{-1} \mathrm{~km} \mathrm{~s}^{-1}$ (Dame et al. 2001) and $1.82 \times 10^{18} \mathrm{~cm}^{-2} \mathrm{~K}^{-1} \mathrm{~km} \mathrm{~s}^{-1}$ (Dickey \& Lockman 1990). The same conversion factors were used to compute the cumulative absorption column at the pulsar's position shown in Fig. 6 (top panel). From this curve, we estimate that, along the line of sight of the pulsar, the $N_{\mathrm{H}}$ between the observer and the back of the VMR is $0.97 \times 10^{22} \mathrm{~cm}^{-2}$, while the total $N_{\mathrm{H}}$ along the line of sight is $1.65 \times 10^{22} \mathrm{~cm}^{-2}$.

Using the previously generated maps, a comparison plot was built (see Fig. 8) where the $N_{\mathrm{H}}$ derived from the gas maps and integrated up to the VMR is plotted against the best-fit values of the X-ray absorption for several positions in the sky. No correlation is observed in this plot indicating that the SNR is lying in the foreground of the VMR. In addition we note that

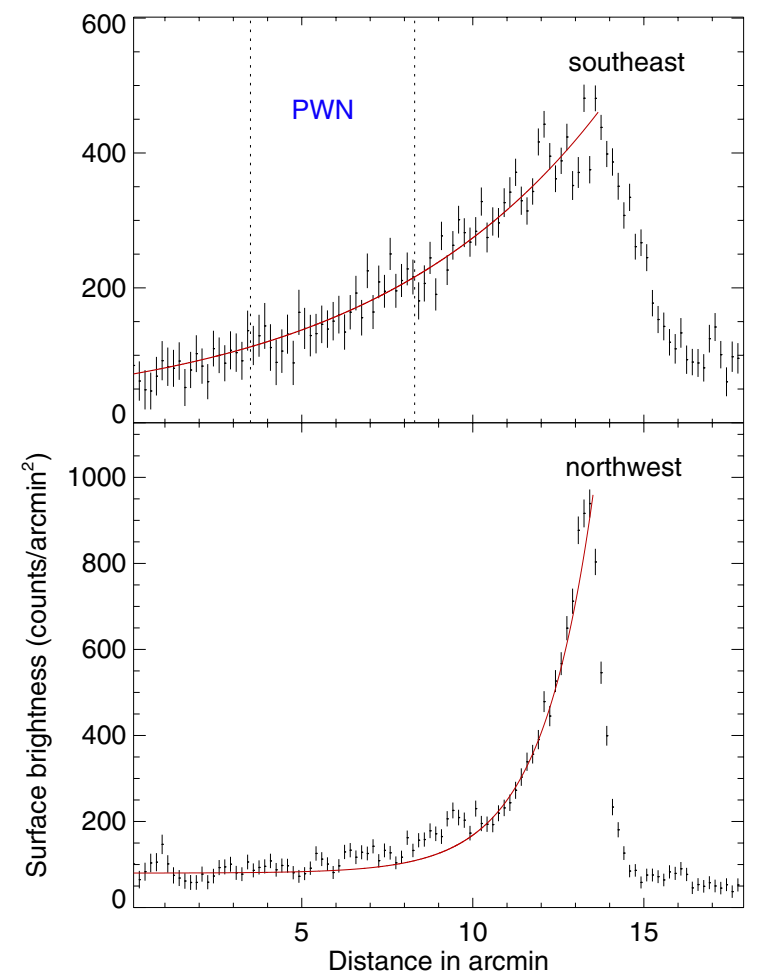

Fig. 5. Radial profiles obtained in the rims of the RX J0852.0-4622 SNR in the southeast (top) and the northwest (bottom) along the spectral extraction regions shown in Figs. 1, 4. In the southeast, the distance of the PWN from the front shock is represented by the dashed lines. The best-fit exponentially decreasing model in the downstream side of the shock is illustrated with the solid lines.

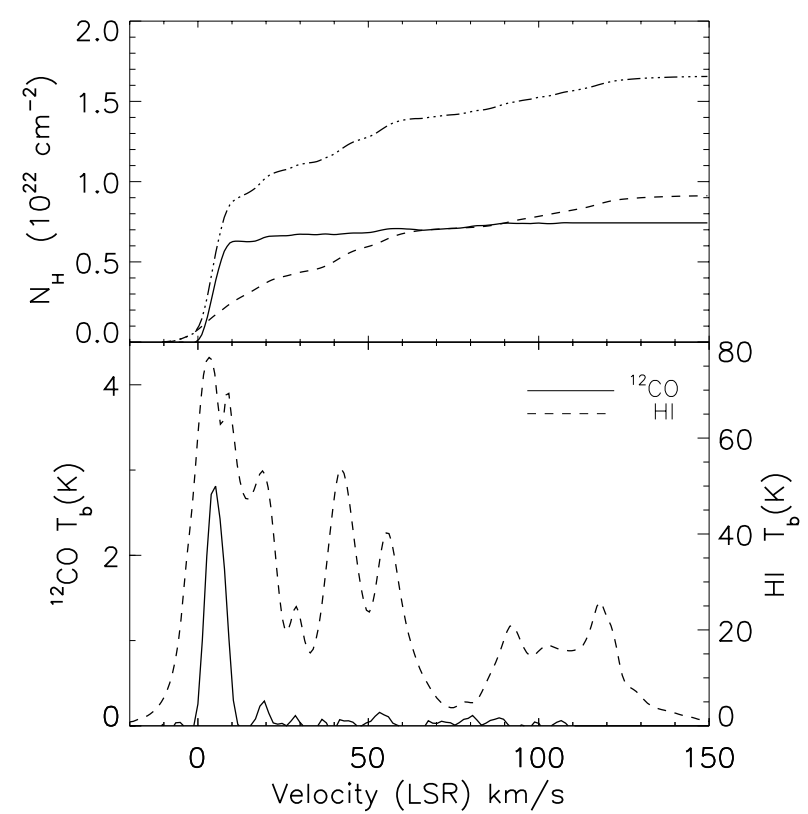

Fig. 6. Top: cumulative absorption column density (dot-dashed line) together with the ${ }^{12} \mathrm{CO}$ and $\mathrm{HI}$ contributions as a function of the radial velocity integrated on a $0.1^{\circ}$ box region centered on the pulsar. Bottom: corresponding ${ }^{12} \mathrm{CO}$ and $\mathrm{HI}$ spectra at the position of the pulsar. The Vela molecular ridge, centered at $V_{\mathrm{LSR}} \sim 5 \mathrm{~km} \mathrm{~s}^{-1}$, is the main ${ }^{12} \mathrm{CO}$ structure along the pulsar's line of sight.

the $N_{\mathrm{H}}$ toward the pulsar $\left(0.64_{-0.11}^{+0.13} \times 10^{22} \mathrm{~cm}^{-2}\right)$ is slightly lower than the column toward the SNR rim in the same region 


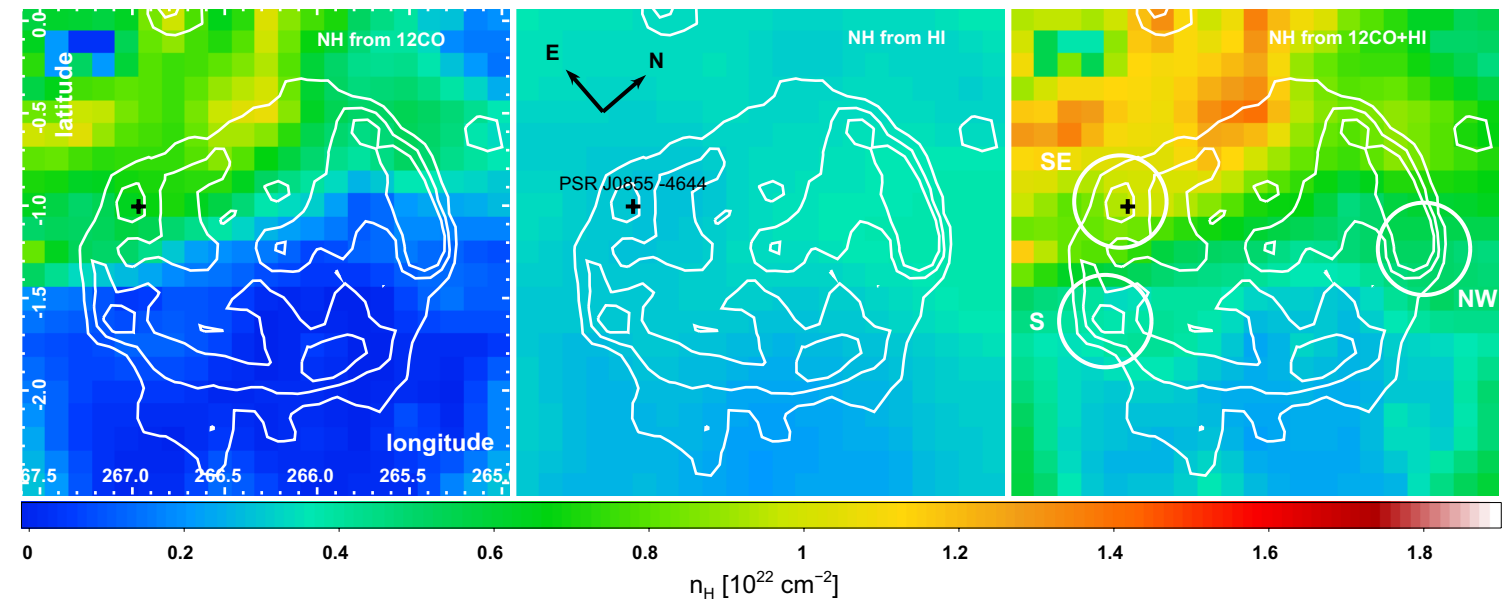

Fig. 7. Maps of the absorption column density, derived from the molecular gas (left panel), the atomic gas (middle panel), and the sum of the two previous components (right panel) when integrating over radial velocities from $-10 \mathrm{~km} \mathrm{~s}^{-1}$ to $15 \mathrm{~km} \mathrm{~s}^{-1}$ (see Sect. 3 for details). This specific velocity range provides an estimate of the absorption column for a source located just behind the Vela molecular ridge. The position of PSR J0855-4644 is shown with a black cross and the XMM-Newton pointings used in this study are represented by the white circles in the right panel. The white contours are taken from a ROSAT high-energy image $(E>1.3 \mathrm{keV})$ and reveal the SNR RX J0852.0-4622.

$\left(0.88_{-0.06}^{+0.08} \times 10^{22} \mathrm{~cm}^{-2}\right)$, suggesting that the pulsar is closer to, or at the same distance as the SNR RX J0852.0-4622.

An additional test to determine whether the pulsar is in the foreground or background of the VMR is to look for the ratio of neutral hydrogen (related to the X-ray absorption column $N_{\mathrm{H}}$ ) to free electrons (given by the pulsar radio dispersion measure DM). As discussed in Gaensler et al. (2004), this ratio has been observed to be high for pulsars that are known to lie behind dense molecular material (e.g. $N_{\mathrm{H}} / \mathrm{DM}=85$ (40) for PSR J1747-2958 and PSR 1757-24), whereas the typical value for other pulsars is on the order of 5-10. The value we derive for PSR J0855-4644 is $8 \pm 1.5$, which suggests that the pulsar is not located behind dense material.

We therefore conclude that both the SNR and the pulsar are lying in the foreground of the VMR, i.e., at a distance $d \leq 900$ pc. This distance constraint to the RX J0852.0-4622 SNR is compatible with a recent estimate based on the measurement of the proper motion of the shock in X-rays in the northwestern part of the rim by Katsuda et al. (2008), who derived $d \sim 750 \mathrm{pc}$. Concerning the pulsar, a closer distance (than the $4 \mathrm{kpc}$ derived from the radio DM measurements) confirms a suggestion made by Redman \& Meaburn (2005), who derived a distance of $\sim 750 \mathrm{pc}$.

\section{Discussion}

\subsection{Proposed scenario}

In the previous sections, we have shown that RX J0852.0-4622 and PSR J0855-4644 are lying in the foreground or at the interface of the VMR. The SNR and the pulsar are therefore thought to be nearby ( $d \leq 900 \mathrm{pc}$ ), but surprisingly, the absorption columns derived from the $\mathrm{X}$-ray analysis show high values ranging from 0.64 to $0.88 \times 10^{22} \mathrm{~cm}^{-2}$. This is intriguing because the $N_{\mathrm{H}}$ toward the Vela SNR, whose distance is known precisely ${ }^{4}$ to be $d=287_{-17}^{+19} \mathrm{pc}$ (Dodson et al. 2003), is much lower at $\sim 10^{20} \mathrm{~cm}^{-2}$.

To understand this apparent discrepancy, we investigated the spatial distribution and the density of the local gas toward the Vela region. We note that due to the intrinsic velocity dispersion

\footnotetext{
4 The distance to the Vela pulsar was measured by parallax.
}

of the VMR emission both in $\mathrm{HI}$ and in ${ }^{12} \mathrm{CO}$ (see Fig. 6, bottom) and the proximity of the object, it is not possible to separate the emission from the VMR from the foreground emission. However, the density of the nearby gas (the Local Bubble) can be probed by other methods such as the study of the absorption line features of $\mathrm{NaI}$ and $\mathrm{CaII}$ toward stars with known distances (see Lallement et al. 2003; Welsh et al. 2010). These studies revealed interstellar tunnels of low density connecting the Local Bubble to other surroundings cavities. One of these tunnels is in the line of sight of the Vela region at $\ell \sim 265^{\circ}$, extending at least to $250 \mathrm{pc}$ (Welsh et al. 2010). This tunnel could explain the low value of the $N_{\mathrm{H}}$ measured in X-rays in the direction of the Vela SNR. Using the $N_{\mathrm{H}}$ measured toward the Vela SNR $\left(10^{20} \mathrm{~cm}^{-2}\right)$, the mean density in this line of sight is on the order of $0.1 \mathrm{~cm}^{-3}$.

After the Local Bubble, the line of sight encounters the Gum nebula, a large-scale structure centered at $\ell=262^{\circ}, b=-3^{\circ}$ and located at a distance of $\sim 500 \mathrm{pc}$ for a physical radius of $\sim 150 \mathrm{pc}$ and an angular radius of $17^{\circ}$. Over a distance of 200 or $600 \mathrm{pc}$ (distance between the Vela SNR at $287 \mathrm{pc}$ and the VMR at $700 \pm 200 \mathrm{pc}$ ), the required densities must be on the order of 9 to $3 \mathrm{~cm}^{-3}$ respectively to reach the $N_{\mathrm{H}}$ value measured in X-rays of $\sim 0.6 \times 10^{22} \mathrm{~cm}^{-2}$ toward PSR J0855-4644. When integrating the HI spectrum between the Earth and the background of the VMR $\left(-10 \mathrm{~km} \mathrm{~s}^{-1}\right.$ to $\left.15 \mathrm{~km} \mathrm{~s}^{-1}\right)$, the HI alone contributes to $0.3 \times 10^{22} \mathrm{~cm}^{-2}$ in the line of sight toward the pulsar, corresponding to a mean density of $2-5 \mathrm{~cm}^{-3}$ over a distance of 600 and $200 \mathrm{pc}$. In addition, the HI emission is homogeneously spatially distributed around the RX J0852.0-4622 SNR region and is not correlated with the VMR, as shown in the HI map shown in Fig. 7 (middle panel), thus contributing to all the lines of sight used in the X-ray analysis, which is consistent with the relatively high $N_{\mathrm{H}}$ values measured across the SNR. The aforementioned densities are compatible with the density of $\sim 7 \mathrm{~cm}^{-3}$ measured in the inner region of the Gum nebula (the thick HI shell discussed in Dubner et al. 1992; Reynoso \& Dubner 1997), which is spatially overlapping our region of interest. $\mathrm{H}_{\alpha}$ observations of the nebula by Reynolds (1976) provided similar values for the densities ( 1 to $5 \mathrm{~cm}^{-3}$ ). One possible origins of the Gum nebula is that the supershell is produced by the combination of stellar winds and repeated SN explosions (McCray \& Kafatos 1987; Reynoso \& Dubner 1997), which makes it a plausible location for PSR J0855-4644 and RX J0852.0-4622. 
Finally, changing the electron density of the Gum nebula in the NE2001 model from $0.5 \mathrm{~cm}^{-3}$ (the currently implemented uniform value for the Gum nebula) to $1 \mathrm{~cm}^{-3}$ is sufficient to reconcile a DM of $239 \mathrm{~cm}^{-3}$ pc with a distance to the pulsar of $900 \mathrm{pc}$. This does not seem unreasonable with regard to the possible variation of density in the Gum nebula.

In summary, the line of sight toward the pulsar first encounters a tunnel of low-density gas $\left(\sim 0.1 \mathrm{~cm}^{-3}\right)$ up to the Vela SNR, then enters the Gum nebula thick HI shell with densities of about $1-5 \mathrm{~cm}^{-3}$. This succession of low- and high-density environment can explain the low $N_{\mathrm{H}}$ toward the Vela SNR and the high $N_{\mathrm{H}}$ toward the RX J0852.0-4622 SNR and the pulsar. While the $\mathrm{HI}$ alone contributes to $0.3 \times 10^{22} \mathrm{~cm}^{-2}$, additional molecular material in the line of sight could contribute to the measured X-ray absorption either in the foreground of the $\mathrm{VMR}^{5}$ or from the external regions of the VMR if the pulsar and SNR are lying at the interface of the molecular cloud.

\subsection{Is pulsar associated with the RX J0852.0-4622 SNR?}

Although the pulsar and the SNR may be at similar distances, an association is unlikely due to the characteristic age of the pulsar $\left(\tau_{\mathrm{c}}=140 \mathrm{kyrs}\right)$ being much larger than the SNR age $\left(t_{\mathrm{SNR}} \sim\right.$ $4000 \mathrm{yrs})$. Assuming that $\tau_{\mathrm{c}}$ could be very different from the real age, the required pulsar's kick velocity to explain its current position would be $\sim 3000 \mathrm{~km} \mathrm{~s}^{-1}$ to travel $12 \mathrm{pc}$ from the SNR center $\left(1^{\circ}\right.$ at $\left.750 \mathrm{pc}\right)$ for an SNR age of 4000 years. This value is much higher than observed in other pulsars (Hobbs et al. 2005). Moreover, for such a high shock speed a bow-shock or a tail structure should appear in the PWN, which is not the case. In addition, a candidate neutron star CXOU J085201.4-461753 (see e.g. Kargaltsev et al. 2002) lies near the geometrical center of the SNR RX J0852.0-4622, which appears as a more simple scenario.

\subsection{TeV emission from the PWN}

With the new distance derived in this study, the ratio $\dot{E} / d^{2}$ for PSR J0855-4644 reaches a value $\geq 10^{36} \mathrm{erg} \mathrm{s}^{-1} / \mathrm{kpc}^{2}$ (for $d \leq 900$ pc and $\dot{E}=1.1 \times 10^{36}$ ergs). According to the TeV $\gamma$-ray PWN population study presented by Carrigan et al. (2008), a high $\dot{E} / d^{2}$ pulsar like this should be detectable by Cherenkov telescopes in $\gamma$-rays. The region of the RX J0852.0-4622 SNR has been extensively studied by the H.E.S.S. experiment and an excess of $\mathrm{TeV} \gamma$-rays is observed at the position of the pulsar (see Fig. 3 in Paz Arribas et al. 2012). However, as the pulsar is in spatial coincidence with the SNR shell, it is not possible, given the spatial resolution of the H.E.S.S. telescopes, to disentangle the contribution from the SNR shell from the contribution of the PWN. In addition, the azimuthal profile of the $\gamma$-ray emission along the SNR shell does not reveal any significant emission increase with regards to the surrounding SNR shell emission at the position of the pulsar (Paz Arribas et al. 2012).

\subsection{Pulsar contribution to observed $e^{-} / e^{+} C R s$}

This new distance estimate of the pulsar strongly differs from the distance estimated with the radio dispersion measure $(d=4 \mathrm{kpc})$ and therefore implies that PSR J0855-4644 is an energetic and nearby pulsar. After the Vela pulsar, it is the most energetic pulsar within $1 \mathrm{kpc}$ and could therefore contribute to the spectrum

\footnotetext{
5 This material can unfortunately not be separated from the VMR in the ${ }^{12} \mathrm{CO}$ spectrum.
}

of cosmic-rays $\mathrm{e}^{-} / \mathrm{e}^{+}$received at Earth (see Delahaye et al. 2010 , for the contribution of nearby pulsars). Obtaining a good knowledge of the nearby pulsar population is a necessary step to understand the rise in positron fraction with increasing energy measured by the PAMELA experiment (Adriani et al. 2009) and confirmed by the Fermi space telescope (Ackermann et al. 2012).

As the pulsar is rather old $\left(\tau_{\mathrm{c}}=140 \mathrm{kyrs}\right)$ and because no traces of the host SNR have been observed, one can reasonably assume that the CR accelerated in the PWN have had time to escape the SNR. The diffusion timescale of these escaping CRs to reach the Earth scales as $t_{\text {prop }}(E) \approx d^{2} / D(E)$ where $D(E)$ is the diffusion coefficient with a typical value of $\sim 3 \times 10^{28}(E / 1 \mathrm{GeV})^{\delta} \mathrm{cm}^{2} / \mathrm{s}$ with a large uncertainty on the energy dependence $\delta=0.3-0.6$ (see Strong et al. 2007, for a review of $\mathrm{CR}$ propagation in the Galaxy). Therefore, $t_{\text {prop }}(E) \approx$ $100 \operatorname{kyrs}(d / 100 \mathrm{pc})^{2}(E / 1 \mathrm{GeV})^{-0.5}$. For a typical value of $\delta=$ 0.5 , neither PSR J0855-4644 nor the Vela pulsar $\left(\tau_{\mathrm{c}}=11 \mathrm{kyrs}\right)$ are thought to contribute to the observed flux of $\mathrm{e}^{-} / \mathrm{e}^{+}$at $10 \mathrm{GeV}$ because the diffusion timescale is 1500 and 280 kyrs (for $d=$ 750 and $290 \mathrm{pc}$ ). By equating $t_{\mathrm{prop}}=\tau_{\mathrm{c}}$ and for $\delta=0.5$, the energy of the particles that could have diffused up to Earth is $1 \mathrm{TeV}$ and $6 \mathrm{TeV}$ for PSR J0855-4644 and the Vela pulsar.

The previous estimates assume that the particles start to diffuse at the birth of the SNR and do not take into account the time for the particles accelerated in the PWN to break through the SNR shell. This escape effect could be not negligible in the case of the Vela pulsar (see Hinton et al. 2011, for a study of the diffusive escape effect on the particle spectrum) due to the relative young age of the system $\left(\tau_{\mathrm{c}}=11 \mathrm{kyrs}\right)$.

\subsection{Comparison of the width of the SNR rims}

In the XMM-Newton observation of the southeast region of the RX J0852.0-4622 SNR, a faint rim of non-thermal emission was detected (see Fig. 1, right). In Sect. 2.7, the downstream thickness of the rim was measured with an exponentially decreasing model. We observed that the filament in the southeast is nearly four times thicker $\left(w_{d}=6.85 \pm 0.55 \mathrm{arcmin}\right)$ than the bright and thin $\left(w_{d}=1.61 \pm 0.05 \mathrm{arcmin}\right)$ filament observed in the northwest of the SNR shown in Fig. 4.

Assuming that the thickness of the rim is limited by the synchrotron losses, while the electrons are advected away from the shock, one can derive a lower limit to the value of the downstream magnetic field $B_{d}$. To derive this value, we adapted Eq. (9) from Parizot et al. (2006) to keep track of the observed photon energy $E_{\mathrm{ph}}$ and obtained the following relation:

$B_{\mathrm{d}} \geq 37 \mu \mathrm{G} \times V_{\mathrm{sh}, 3}^{2 / 3} \times\left(\theta_{\mathrm{obs}, \operatorname{arcmin}} \times d_{\mathrm{kpc}}\right)^{-2 / 3} \times E_{\mathrm{ph}, \mathrm{keV}}^{-1 / 3}$,

where $V_{\mathrm{sh}, 3}$ is the shock speed in units of $1000 \mathrm{~km} \mathrm{~s}^{-1}, \theta_{\mathrm{obs} \text {,arcmin }}$ is the observed width of the rim at half maximum in units of arc minutes $\left(\theta_{\mathrm{obs}}=w_{\mathrm{d}} \times \log (2)\right)$ and $E_{\mathrm{ph}}$ is the average emitted photon energy in keV. For a distance to the RX J0852.0-4622 SNR of 750 pc (see Katsuda et al. 2008, and Sect. 3 of this article for a more detailed discussion of the SNR distance), a shock speed of $3000 \mathrm{~km} \mathrm{~s}^{-1}$ (Katsuda et al. 2008) and a mean photon energy of $\sim 2.5 \mathrm{keV}$, the downstream magnetic field value is $24 \mu \mathrm{G}$ and $63 \mu \mathrm{G}$ for the southeast and for the northwest rim.

We note that in the northwest region, an X-ray proper motion of the shock $\left(\dot{\theta}_{\mathrm{sh}}=V_{\mathrm{sh}} / d\right)$ has been measured ${ }^{6}$ by Katsuda et al. (2008). As the ratio of $V_{\mathrm{sh}} / d$ can be replaced by $\dot{\theta}_{\mathrm{sh}}$ in Eq. (1), the

6 The value of $V_{\mathrm{sh}}$ and $d$ used in the previous calculation were derived from the aforementioned proper motion measurements. 


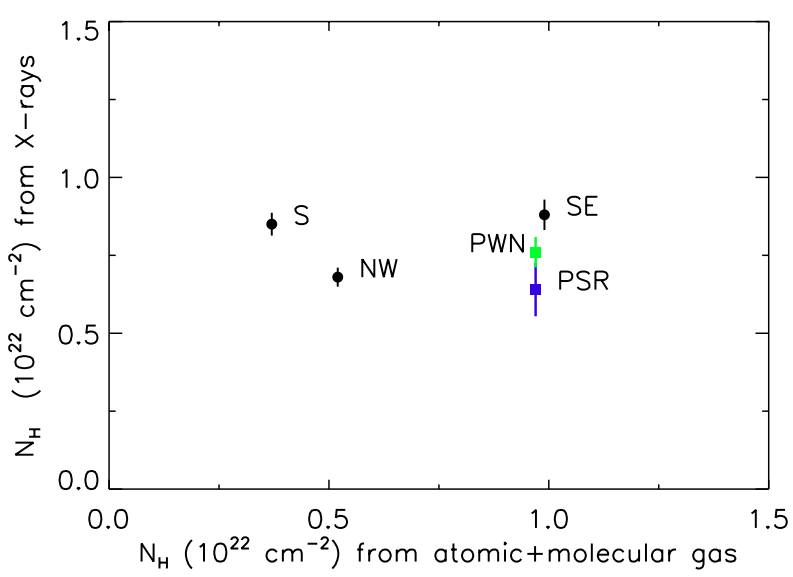

Fig. 8. Comparison plot of the absorption column density values derived from the atomic and molecular gas and from the $\mathrm{X}$-rays using XMM-Newton observations. The X-ray columns derived toward the rims of the RX J0852.0-4622 SNR, PSR J0855-4644 and the PWN are represented by black circles, a blue square, and a green square. The errors on the X-ray $N_{\mathrm{H}}$ are $1 \sigma$ error bars. The label over each point corresponds to regions where the values were measured as defined in Fig. 7.

estimate of the magnetic field, at least in the northwest, is in fact independent of the hypothesis on the shock speed and distance.

The magnetic field downstream of the shock is therefore significantly different in these two regions. This emphasizes that the magnetic field derived from a one-zone modeling of the SED of the whole SNR (giving an average value of the magnetic field) is not directly comparable to the magnetic field derived from a filament in a specific region of the SNR.

\section{Conclusions}

The search for the X-ray counterpart of PSR J0855-4644 using $X M M-N e w t o n$ led to the following conclusions:

1. A point-like X-ray counterpart has been discovered in spatial coincidence with the position of the radio pulsar and a surrounding extended emission of non-thermal origin was found. The nebula has a maximum extent of about $150^{\prime \prime}$ and the azimuthal profile has revealed two knots of emission (with angular separation of $\sim 180^{\circ}$ ), suggesting possible jets around the pulsar. High spatial resolution observations with Chandra might reveal the fine structure of the nebula.

2. A faint rim of non-thermal emission representing the southeastern part of the RX J0852.0-4622 SNR was detected. The filament in this region is thicker than the bright and thin X-ray filaments observed in the northwest of the SNR. Assuming that the thickness of the rim is dominated by the synchrotron losses, the downstream magnetic field is $24 \mu \mathrm{G}$ and $63 \mu \mathrm{G}$ respectively for the southeast and for the northwest rim. These significantly different values might indicate different acceleration and/or environmental conditions at the shock around the SNR.

3. By comparing the X-ray column density on the pulsar and on different regions of the SNR with the gas map (using ${ }^{12} \mathrm{CO}$ and $\mathrm{HI}$ emission as tracers), we concluded that both the RX J0852.0-4622 SNR and PSR J0855-4644 are in the foreground or at the interface of the Vela Molecular Ridge, resulting in an upper limit of $d \leq 900 \mathrm{pc}$. Although the pulsar and the SNR may be at similar distances, they are not likely to be associated.

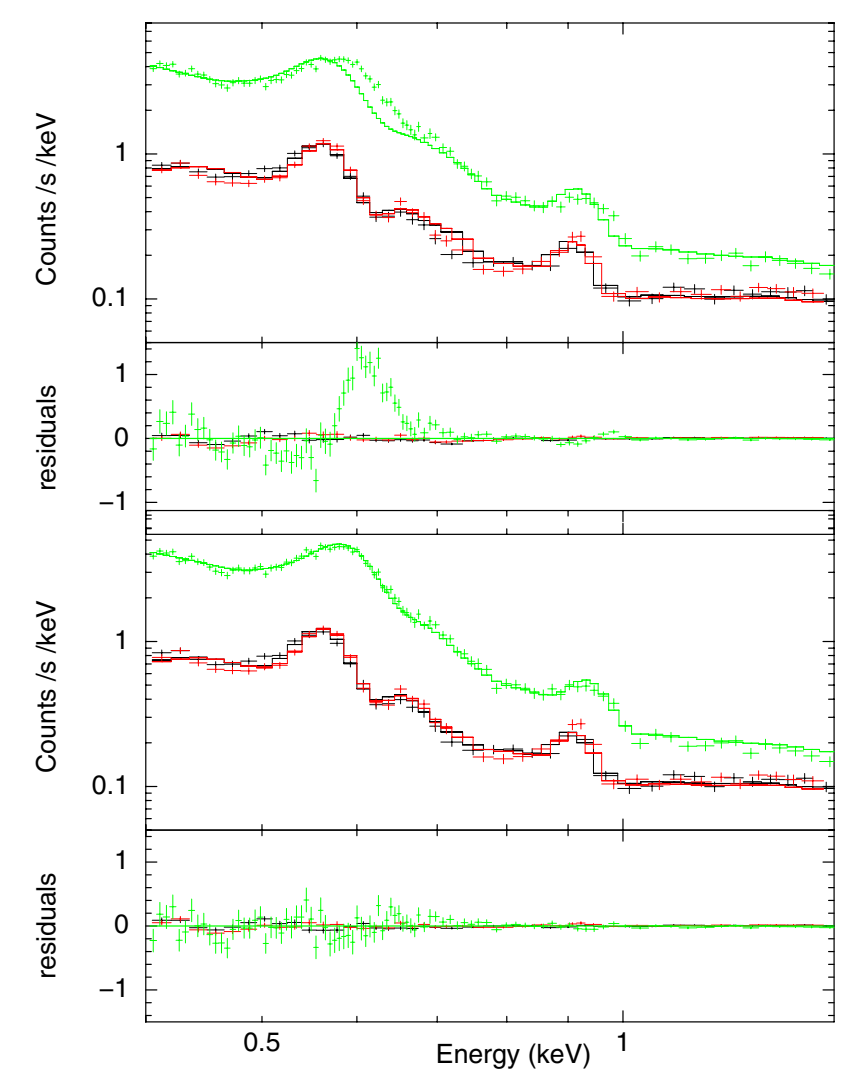

Fig. A.1. Top: X-ray spectrum of the nebula extracted from a $15-150^{\prime \prime}$ annulus region shown in Fig. 2 focused on the thermal emission with MOS1, MOS2 and PN spectra represented in black, red, and green. The same spectral model as in Fig. 3 is overlaid. Bottom: same spectra as in the top panel but with the PN response matrix modified by the gain response model implemented in Xspec.

4. The new estimate of the pulsar distance completes our knowledge of the nearby pulsar population. If the distance of the pulsar is confirmed for instance through radio parallax measurements, PSR J0855-4644 would be the second most energetic pulsar (after the Vela pulsar) within $1 \mathrm{kpc}$ and may contribute to the observed $\mathrm{e}^{-} / \mathrm{e}^{+}$spectrum.

Acknowledgements. F.A. would like to thank Isabelle Grenier for discussions on the nearby interstellar medium and David Smith for useful discussion on the NE2001 Galactic electron distribution model. The help from Konrad Dennerl is also greatly appreciated to solve the calibration, problems with the XMM-Newton PN camera.

\section{Appendix A: Calibration issues with the PN camera}

The thermal emission from the Vela SNR is extremely bright at low energies $(E<1 \mathrm{keV})$, an energy range where the PN camera is more sensitive than the MOS. With this statistics we are limited by our knowledge of the camera calibration. Here we have observed a deficiency in the low-energy calibration of the spectral response of the PN camera. This effect is most visible around $0.6 \mathrm{keV}$ in Fig. A.1, where the oxygen lines cannot be reproduced by the APEC thermal model.

This is a known calibration problem ${ }^{7}$ that is being investigated by the XMM-Newton PN calibration team. There are no

\footnotetext{
7 More information on this calibration issue can be found here: http: //www2.le.ac.uk/departments/physics/research/ src/Missions/xmm-newton/technical/cal-meetings\# madrid-0310
} 
known similar calibration problems for the MOS instruments. Following their recommendation, we allowed for an empirical shift in energy in the PN response file using the gain response model implemented in Xspec. The fitted energy shift is on the order of $15 \mathrm{eV}$. Similar best-fit parameters where obtained when fitting independently the MOS and response-corrected PN spectra. This confirms that the correction of the response matrix does not alter the physical parameters that we derive from the spectrum. In the spectral analysis of the article we have therefore used the MOS1, MOS2, and response-corrected PN spectra.

\section{References}

Ackermann, M., Ajello, M., Allafort, A., et al. 2012, Phys. Rev. Lett., 108, 011103

Adriani, O., Barbarino, G. C., Bazilevskaya, G. A., et al. 2009, Nature, 458, 607 Aharonian, F., Akhperjanian, A. G., Bazer-Bachi, A. R., et al. 2007, ApJ, 661, 236

Aschenbach, B. 1998, Nature, 396, 141

Aschenbach, B., Egger, R., \& Trümper, J. 1995, Nature, 373, 587

Aschenbach, B., Iyudin, A. F., \& Schönfelder, V. 1999, A\&A, 350, 997

Bamba, A., Yamazaki, R., \& Hiraga, J. S. 2005, ApJ, 632, 294

Carrigan, S., Hinton, J. A., Hofmann, W., et al. 2008, in Intern. Cosmic Ray Conf., 2, 659

Carter, J. A., \& Read, A. M. 2007, A\&A, 464, 1155

Cordes, J. M., \& Lazio, T. J. W. 2002 [arXiv: 0207156]

Dame, T. M., Hartmann, D., \& Thaddeus, P. 2001, ApJ, 547, 792

De Luca, A., \& Molendi, S. 2004, A\&A, 419, 837

Delahaye, T., Lavalle, J., Lineros, R., Donato, F., \& Fornengo, N. 2010, A\&A, 524, A51

Dickey, J. M., \& Lockman, F. J. 1990, ARA\&A, 28, 215

Dodson, R., Legge, D., Reynolds, J. E., \& McCulloch, P. M. 2003, ApJ, 596, 1137
Dubner, G., Giacani, E., Cappa de Nicolau, C., \& Reynoso, E. 1992, A\&AS, 96, 505

Gaensler, B. M., van der Swaluw, E., Camilo, F., et al. 2004, ApJ, 616, 383

Hinton, J. A., Funk, S., Parsons, R. D., \& Ohm, S. 2011, ApJ, 743, L7

Hobbs, G., Lorimer, D. R., Lyne, A. G., \& Kramer, M. 2005, MNRAS, 360, 974

Kalberla, P. M. W., McClure-Griffiths, N. M., Pisano, D. J., et al. 2010, A\&A, 521, A17

Kargaltsev, O., \& Pavlov, G. G. 2008, in 40 Years of Pulsars: Millisecond Pulsars, Magnetars and More, eds. C. Bassa, Z. Wang, A. Cumming, \& V. M. Kaspi, AIP Conf. Ser., 983, 171

Kargaltsev, O., Pavlov, G. G., Sanwal, D., \& Garmire, G. P. 2002, ApJ, 580, 1060

Katsuda, S., Tsunemi, H., \& Mori, K. 2008, ApJ, 678, L35

Kramer, M., Bell, J. F., Manchester, R. N., et al. 2003, MNRAS, 342, 1299

Lallement, R., Welsh, B. Y., Vergely, J. L., Crifo, F., \& Sfeir, D. 2003, A\&A, 411,447

Liseau, R., Lorenzetti, D., Nisini, B., Spinoglio, L., \& Moneti, A. 1992, A\&A, 265,577

McClure-Griffiths, N. M., Pisano, D. J., Calabretta, M. R., et al. 2009, ApJS, 181,398

McCray, R., \& Kafatos, M. 1987, ApJ, 317, 190

Mitra, D., \& Ramachandran, R. 2001, A\&A, 370, 586

Murphy, D. C., \& May, J. 1991, A\&A, 247, 202

Parizot, E., Marcowith, A., Ballet, J., \& Gallant, Y. A. 2006, A\&A, 453, 387

Paz Arribas, M., Schwanke, U., Sushch, I., et al. 2012, Proc. 32nd ICRC, 7, 140 [arXiv: 1203.2532]

Redman, M. P., \& Meaburn, J. 2005, MNRAS, 356, 969

Reynolds, R. J. 1976, ApJ, 203, 151

Reynoso, E. M., \& Dubner, G. M. 1997, A\&AS, 123, 31

Strong, A. W., Moskalenko, I. V., \& Ptuskin, V. S. 2007, Ann. Rev. Nuclear Part. Sci., 57, 285

Wallerstein, G., Jenkins, E. B., \& Silk, J. 1980, ApJ, 240, 834

Welsh, B. Y., Lallement, R., Vergely, J.-L., \& Raimond, S. 2010, A\&A, 510, A54

Wilms, J., Allen, A., \& McCray, R. 2000, ApJ, 542, 914 